
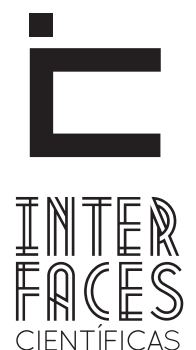

EDUCAÇÃO

ISSN IMPRESSO 2316-333X

ISSN ELETRÔNICO 2316-3828

PARTE 2 - FORMAÇÃO E ATUAÇÃO PROFISSIONAL DA EDUCAÇÃO SOCIAL

\title{
PRÁCTICA PROFESIONAL Y FORMACIÓN DEL EDUCADOR SOCIAL EN ESPAÑA
}

Ángel De-Juanas Oliva ${ }^{1}$

María Rosario Limón Mendizábal ${ }^{2}$
Miguel Melendro Estefanía ${ }^{3}$

\section{RESUMEN}

La Pedagogía Social es una de las disciplinas de las ciencias sociales y humanas, y es también, una profesión social en la que el educador o educadora social es reconocida como figura y perfil profesional específico. Y es una importante área de especialización educativa en las universidades europeas. Este artículo aporta un análisis y describe las etapas de su desarrollo, centradas principalmente en el ámbito profesional y la formación del Educador Social en España. Esto lo aborda tanto desde la lógica disciplinar como desde la lógica profesional. Así mismo, aporta una reflexión sobre las competencias necesa- rias del Educador Social. El enfoque por competencias aparece con fuerza en el campo de la formación en la medida en que viene vinculado a las prácticas socioeducativas, al trabajo pedagógico de los problemas educativos en las diferentes situaciones y a los proyectos que desean materializar, los educadores, en sus espacios de trabajo.

\section{PALABRAS-CLAVE}

Educación Social. Competencias. Ámbitos Laborales. Espacio Europeo de Educación Superior. Pedagogía Social. 


\section{RESUMO}

A Pedagogia Social é uma das disciplinas das ciências sociais e humanas e é, também, uma profissão social na qual o educador ou educadora social é reconhecido com um perfil profissional específico. E é uma importante área de especialização educativa nas universidades europeias. Este artigo faz uma análise e descreve as etapas do seu desenvolvimento, centradas principalmente no âmbito profissional e a formação do Educador Social na Espanha. Abordará tanto a lógica disciplinar como a lógica profissional. Também, aborda uma reflexão sobre as competências necessárias do Educador Social.
O enfoque por competências aparece com força no campo da formação na medida em que se vincula às práticas socioeducativas, ao trabalho pedagógico dos problemas educativos nas diferentes situações e aos projetos que desejam instrumentalizar os educadores em seus espaços de trabalho.

\section{PALAVRAS-CHAVE}

Educação Social. Competências. Ambiente de TrabaIho. Espaço Europeu de Educação Superior. Pedagogia Social.

\section{ABSTRACT}

The Social Pedagogy is one of the disciplines of the humanities and social sciences and is also a social profession in which the educator or social educator is recognized as a specific professional profile. It is an important area of educational specialization in European universities. This article provides an analysis and describes the periods on their development, focused mainly on the professional field and the Social Educator's training in Spain. This is addressed from the disciplinary logic as much as from the professional logic. It also makes a reflection about the competences needed to be a Social Educator. The focus on competencies appears hard on the training field in that it is linked to social and educational practices, the pedagogical work of educational problems in different situations and projects wishing to equip educators in their workspaces.

\section{KEYWORDS}

Social Education. Competences. Workplace. European Higher Education Area. Social Pedagogy. 


\section{INTRODUCCIÓN}

La importante experiencia acumulada sobre la práctica profesional y la formación del educador social en España, es sin duda una referencia interesante para entender esta profesión y sus posibilidades de desarrollo en el seno de las sociedades del nuevo milenio.

Desde sus orígenes, hasta su actual y cada vez más extendida presencia social, se tratan en las siguientes páginas una serie de cuestiones de sumo interés: los modelos que sirvieron de base para su implantación en nuestro país, las circunstancias políticas y sociales que la propiciaron, las conexiones entre formación universitaria y desarrollo profesional, el impacto del EEES en la formación de los educadores sociales $\mathrm{y}$, de forma más detallada, las competencias, ámbitos y espacios profesionales del educador y la Educación Social en nuestro país.

Se podría decir, con Ortega, Caride y Úcar (2013, p. 9-12) que "la Pedagogía Social y la Educación Social en España son el resultado de tres corrientes de pensamiento y acción que llegan a nuestro país a lo largo del siglo XX", y que orientan tanto a la formación como a la profesionalización de educadores/as sociales.

La primera de ellas, la corriente francófona, iniciada en este país como respuesta a las necesidades de su infancia y juventud tras la finalización de la Segunda Guerra Mundial, se caracterizada por su fuerte implicación social y política, el trabajo sociocomunitario y la educación especializada con la infancia y juventud inadaptada socialmente. Podemos situar su origen en Francia, en 1947, con la creación de la Asociación Nacional de Educadores de Jóvenes Inadaptados (ANEJI), que extendió sus actuaciones a nivel internacional - Estado Unidos, Canadá, Holanda, Italia, Bélgica, Alemania- y en 1951 dio paso a la creación de la Asociación Internacional de Educadores de Jóvenes Inadaptados (AIEJI) y al reconocimiento de la figura profesional del Educador Especializado, antecedente a la vez que figura coetánea de la del Educador Social (CHAMSEDDINE, 2013).

La segunda corriente descrita por estos autores es la corriente alemana, de marcado carácter teórico-filosófico, que llega a nuestro país a principios de siglo y renace con fuerza también tras la última contienda mundial. Esta línea de trabajo "se instaura fundamentalmente en las universidades, constituyendo el núcleo que conforma el pensamiento universitario inicial sobre la Pedagogía Social" (ORTEGA, CARIDE y ÚCAR, 2013, p. 9) y será origen de una formación superior polivalente que unifica las aportaciones de la Pedagogía Social, las Ciencias de la Educación y el Trabajo Social.

Por último, la corriente anglosajona, más centrada en las intervenciones psicosociales que socioeducativas, ha tenido aparentemente una incidencia menos directa en el proceso de profesionalización del educador social. Sin embargo sus aportaciones metodológicas - desde la Psicología, la Psicopedagogía y el Trabajo Social fundamentalmente - y su influencia en las técnicas y estrategias de acción socioeducativa son notables, además de la cada vez mayor incidencia en ellas de las investigaciones procedentes de este enfoque.

Cabe señalar por otra parte, con Chamseddine (2013, p. 3), como el educador recibe diversas denominaciones en diferentes países europeos, y cómo son distintas también sus funciones y escenarios de intervención. Así, en España y Bélgica recibe la denominación de Educador Social; Educador Especializado en Luxemburgo, Holanda y Suiza; Animador y/o Educador Especializado en Francia; Trabajador Social de Internado, Trabajador para los Jóvenes y la Comunidad, en Reino Unido; Pedagogo Social en Alemania; Educador Profesional en Italia; Educador Social o Pedagogo en Portugal. 


\section{ORIGEN Y DESARROLLO PROFESIONAL DEL EDUCADOR SOCIAL EN ESPAÑA}

En nuestro país, el frecuentado ámbito de los servicios socioeducativos para la familia, la infancia y la juventud nos aporta un claro ejemplo sobre la construcción profesional de la figura del educador/a social. En él se verá aparecer, a partir de la década de los sesenta, y muy influenciado por la corriente francófona, una serie de nuevas profesiones "sociales" de la educación. Junto a los educadores de centros residenciales de menores, de protección y de reforma, otros profesionales de la educación en el denominado "medio abierto" se ocuparon de la atención y la prevención de situaciones de exclusión social infantil y juvenil.

Desde entonces, y hasta finales de los noventa, "asistimos a una transición de la figura del "educador especializado" - algunos le añaden "en marginación" - a las del educador/a de calle o el educador/a familiar, para llegar finalmente a la consolidación de la figura profesional del educador/a social" (MELENDRO, 1999). Una figura profesional que aglutinará no solo al sector mayoritario de los educadores procedentes de los servicios sociales, esencialmente de los dedicados a la familia, infancia y juventud, sino también a aquellos que trabajaban en otros ámbitos, como la animación sociocultural, la educación de adultos o la atención a mayores o a personas con discapacidad.

Esta diversidad de perspectivas, ámbitos y figuras profesionales, previa a la unificación del campo a través de la Educación Social, se corresponderá históricamente con la transición política en el país y un amplio periodo de crecimiento económico y de construcción del Estado de bienestar. Como señalará Caride (2011, p. 45), refiriéndose a los años ochenta en nuestro país,

Este proceso histórico sólo puede entenderse adecuadamente si se analiza la estrecha relación que mantiene con el desarrollo de la cultura democrática y la intensa modernización que se experimenta en todos los ámbitos de la vida social española. También, e indiscutiblemente, en la educación y en la apertura de sus teorías y prácticas a nuevos modos de educar y educarse en sociedad. En este sentido, deben significarse los compromisos que ha adquirido la Pedagogía Social con los procesos de transformación social, en la mejora de la calidad de vida y en el bienestar de la ciudadanía.

En ese periodo de consolidación paulatina de la Educación Social con mayúsculas, que se extiende de los años sesenta al inicio de los dos mil, no serán pocos los problemas y entre ellos, de forma destaca$\mathrm{da}$, las dificultades corporativas y de competencia con otras profesiones de los social que también entonces abrían sus espacios en estos ámbitos de intervención. Especialmente psicólogos, trabajadores sociales, maestros, animadores socioculturales, sociólogos o abogados. Sin embargo la necesidad de trabajar desde equipos multiprofesionales realidades complejas y de difícil abordaje, y los buenos resultados de las actuaciones coordinadas de tipo interdisciplinar e institucional, fueron ayudando a construir un tejido profesional cada vez más compacto y efectivo. Si bien aún hay mucho que avanzar en este sentido, el trabajo en equipos interdisciplinares se ha ido imponiendo y es ya una forma de hacer habitual en los espacios de acción socioeducativa.

Para amparar y fortalecer la figura del educador social, a partir de los años ochenta aparecieron las primeras asociaciones de educadores/as sociales, organizadas territorialmente en las diferentes comunidades autónomas. A partir de ellas y de su actividad de divulgación, intercambio de experiencias y reconocimiento profesional, se fueron constituyendo diferentes redes, cada vez más consistentes y activas. Así se avanzó en la creación de los Colegios oficiales de educadores sociales, extendidos por todo el país y unificados en el Consejo General de Colegios Oficiales de Educadores Sociales (CGCEES) a partir de 2007. Paralelamente, el ámbito académico se organiza también con la constitución, en septiembre de 
2000, de la Sociedad Iberoamericana de Pedagogía Social (SIPS). Ambas organizaciones sustentan una importante actividad a nivel nacional e internacional, tanto de representación como de divulgación e intercambio, a través de congresos, seminarios y de sus propias publicaciones periódicas, como Pedagogía Social, Revista Interuniversitaria, o la Revista de Educación Social.

Simultáneamente, a través de la actividad y creciente influencia de estas entidades profesionales, junto al interés de la propia Administración y el apoyo de algunos sectores de las universidades españolas, especialmente desde las Facultades de Educación y/o Pedagogía (LIMÓN, 1992), se consigue finalmente la aprobación, por el Real Decreto 1420/1991, de 30 de agosto, del Título Universitario Oficial de Diplomado en Educación Social. En esta normativa se ofrece una definición del Educador Social como "un educador en los campos de la educación no formal, educación de adultos (incluidos los de tercera edad), inserción social de personas desadaptadas y minusválidas, así como la acción socioeducativa".

En la trayectoria narrada, nos reconocemos en la afirmación de Pérez Serrano cuando señala que

\begin{abstract}
La profesión del educador social se ha ido construyendo a lo largo del tiempo a través del buen hacer de los educadores sociales, quienes han mostrado sus competencias y habilidades en las diversas tareas desempeñadas. La sistematización de estas tareas ha contribuido a desarrollar nuevos conocimientos a la vez que han aportado soluciones creativas a los problemas sociales, lo que ha propiciado su progresivo reconocimiento profesional. (PÉREZ SERRANO, 2004, p. 147).
\end{abstract}

\section{3 ÁMBITOS Y ESPACIOS PROFESIONALES DEL EDUCADOR Y EDUCADORA SOCIAL: ANÁLISIS DE COMPETENCIAS}

La Educación Social tiene como referentes básicos científicos a la Pedagogía Social, la Psicología, la Sociología, la Antropología y la Filosofía. Estos referentes aportan elementos teóricos, metodológicos y/o técnicos para el trabajo socioeducativo. Las propuestas de definición de Educación Social del Consejo General de Colegios de Educadoras y Educadores Sociales se refiere a la acción socioeducativa como el acceso a los derechos de ciudadanía y a la promoción social y cultural, en coherencia con la práctica profesional y la reflexión académica de la Pedagogía Social. En la actualidad, las líneas estratégicas de las políticas sociales de la Unión Europea, y de los estados miembros, señalan la inclusión y el aprendizaje a lo largo de la vida entre sus fines y ejes vertebradores. El área de política social en Europa se llama de Empleo, Asuntos Sociales e Inclusión; en España, por ejemplo, tenemos el Plan Nacional de Acción para la Inclusión Social del Reino de España 2013-2016 como referencia de la acción social. Podemos destacar cuatro ámbitos profesionales referidos a la Educación Social que son:

- La Educación Social Especializada, orientada a situaciones de marginación, desadaptación y exclusión social, con la finalidad de promover la prevención y la inclusión social de personas y colectivos de población que viven en estos contextos.

- La Educación de Personas Adultas y Mayores, que atiende a las necesidades socioeducativas de la población adulta vinculadas a su desarrollo personal, su adaptación sociolaboral, el envejecimiento activo y la vida saludable (FERNÁNDEZ y LIMÓN, 2012).

- La Animación Sociocultural, que presta atención a las necesidades socioculturales y de desarrollo comunitario, especialmente significativo dentro de la pedagogía social.

- La Inserción Sociolaboral con colectivos en riesgo de exclusión, que algunas propuestas epistemológicas las sitúan como ámbito propio de trabajo de la Educación Social. Este campo profesional va adquiriendo cada vez una mayor importancia. 
Los estudios de inserción laboral de titulados muestran que los educadores y educadoras sociales suelen incorporarse a los ámbitos profesionales de: 1) Familia, infancia y adolescencia, 2) Atención a la comunidad y 3) Trabajo con colectivos específicos (jóvenes, mayores, mujeres etc.).

En cuanto a los escenarios y espacios educativos, la inserción laboral de los educadores sociales puede realizarse de modo más específico si atendemos a perfiles relacionados con la Educación Social Especializada, podemos identificar distintos centros residenciales para la infancia y juventud, programas de servicios sociales (educación de familia, de calle etc.), programas y dispositivos para la inserción laboral de personas en desventaja social, centros ocupacionales y de intermediación sociolaboral, centros y servicios de acogidas de mujeres, inmigrantes y/o refugiados, pisos tutelados, centros de atención a la drogodependencia, programas de atención a reclusos (medidas alternativas a la prisión, reinserción, ... y un amplio espectro de servicios y programas de atención social y de prevención.

Si atendemos a los perfiles más especializados en Adultos y Mayores nos encontramos con distintos espacios profesionales: Hogar del jubilado/pensionista. Centros de mayores. Centros de día. Residencias. Centros sanitarios. Aulas de tercera edad. Centros cívicos. Escuelas de adultos. Universidades populares. Programas universitarios de mayores, etc.

\subsection{NECESIDAD DE FORMAR EN COMPETENCIAS AL EDUCADOR Y EDUCADORA SOCIAL}

En la actualidad, hablar de formación es casi imposible sin referirse a la noción de competencia. Desde el último cambio de siglo, la preocupación internacional por la reforma de los sistemas educativos y de capacitación de profesionales ha impregnado de competencias todas las titulaciones académicas universitarias, ciclos formativos o formación de postgrado. Las competencias están presentes en todas las titulaciones relacionadas con la Pedagogía Social y Educación Social.
Las competencias son conjuntos complejos de conocimientos, actitudes, valores, emociones y motivaciones que cada persona o cada grupo pone en acción en un contexto concreto para hacer frente a las demandas específicas de cada situación (BERMEJO et al., 2013, p. 3).

La competencia no sólo es conocimiento o habilidad puesto que involucra nuestra habilidad de enfrentar demandas complejas, desarrollar actividades y proyectos de manera satisfactoria en contextos complejos y cambiantes. En este escenario hablar de las competencias de los profesionales de la educación social es reflexionar acerca de las dimensiones cognitivas y no cognitivas (conocimientos, habilidades cognitivas, habilidades prácticas, actitudes, motivaciones, valores y emociones) que afectan a nuestro desempeño profesional. Es hablar de la necesaria movilización de todas nuestras destrezas, para pensar y actuar con la mayor madurez profesional, intelectual y moral.

Las competencias las podemos clasificar en tres grandes tipos de saberes:

1.- Los saberes teóricos o conceptuales, relativos a la información y conocimiento científico, así como a la gestión de los mismos. SABER QUE.

2.- Los saberes prácticos o procedimentales, contextualizados y reflexivos relativos a las formas de actuación, a las destrezas cognitivas (pensamiento crítico e investigador, visión global, organización y planificación), destrezas relacionales o de comunicación, así como destrezas prácticas y manuales (intervenciones profesionales, trabajo en equipo interdisciplinar, recursos y entorno, ...). HACER Y SABER SOBRE ESTE HACER.

3.- Los saberes actitudinales y éticos, relativos a las características personales y a otros aspectos que consideramos esenciales que nos comprometen con los usuarios, los compañeros, los equipos y las organizaciones (valores éticos, actitudes, etc...). SABER SER, ESTAR, PARA QUÉ Y POR QUÉ. 


\subsection{COMPETENCIAS PROPUESTAS POR LOS EDUCADORES SOCIALES}

Señala Sáez Carreras (2009, p. 15) que los educadores sociales, son los que definen, construyen, proponen, sostienen y legitiman, en su combate con otras profesiones y en su abordaje de las cuestiones educativas, aquellas competencias consideradas propias y específicas de la profesión; las necesidades para las que la profesión emergió y fue haciéndose presente en el escenario social, cultural, económico y político en el que se mueve. Para la respuesta a determinadas necesidades educativas, de ayer y de hoy, los educadores sociales consideran necesario tener adquiridas las siguientes competencias propuestas por la Asociación Española de Educadores Sociales (ASEDES) que se recogen en la siguiente tabla:

\begin{tabular}{|l|l|}
\hline \multicolumn{2}{|c|}{\begin{tabular}{c}
\multicolumn{1}{|c|}{ COMPETENCIAS DE LOS EDUCADORES } \\
SOCIALESFUNCIÓN COMPETENCIA
\end{tabular}} \\
\hline FUNCIÓN & COMPETENCIA \\
\hline $\begin{array}{l}\text { Transmisión, formación, } \\
\text { desarrollo y promoción de } \\
\text { la cultura. }\end{array}$ & $\begin{array}{l}\text { - Saber reconocer los bienes culturales de valor social4. } \\
\text { - Dominio de las metodologías educativas y de formación. } \\
\text { - Dominio de las metodologías de asesoramiento y orientación. } \\
\text { - Capacidad para particularizar las formas de transmisión cultural a la singularidad de } \\
\text { los sujetos de la educación } \\
\text { - Dominio de las metodologías de dinamización social y cultural. } \\
\text { - Capacidad para la difusión y la gestión participativa de la cultura. }\end{array}$ \\
\hline $\begin{array}{l}\text { Generación de redes so- } \\
\text { ciales, contextos, proce- } \\
\text { sos y recursos educativos } \\
\text { y sociales. }\end{array}$ & $\begin{array}{l}\text { - Pericia para identificar los diversos lugares que generan y posibilitan un desarrollo } \\
\text { - Conocimiento y destreza para crear y promover redes entre individuos, colectivos e } \\
\text { instituciones. } \\
\text { - Capacidad para potenciar las relaciones interpersonales y de los grupos sociales. } \\
\text { - Capacidad de crear y establecer marcos posibilitadores de relación educativa parti- } \\
\text { cularizados. } \\
\text { - Saber construir herramientas e instrumentos para enriquecer y mejorar los procesos } \\
\text { educativos. } \\
\text { - Destreza para la puesta en marcha de procesos de dinamización social y cultural }\end{array}$ \\
\hline
\end{tabular}

4. Bienes de valor social entendidos en la línea propuesta por el Informe de la UNESCO “La educación encierra un tesoro": ético y cultural artístico-científico/tecnológico y económico/social.

5. Sujetos de la educación se refiere a los individuos, grupos o comunidades que intervienen en la acción socioeducativa.

6. Desarrollo cultural de las comunidades y los colectivos. 


\begin{tabular}{|c|c|}
\hline $\begin{array}{l}\text { Mediación social, cultural } \\
\text { y educativa. }\end{array}$ & $\begin{array}{l}\text { - Conocimientos teóricos y metodológicos sobre mediación en sus diferentes acep- } \\
\text { ciones. } \\
\text { - Destreza para reconocer los contenidos culturales, lugares, individuos o grupos a } \\
\text { poner en relación. } \\
\text { - Dar a conocer los pasos o herramientas de los procesos en la propia práctica. } \\
\text { - Saber poner en relación los contenidos, individuos, colectivos e instituciones. }\end{array}$ \\
\hline $\begin{array}{l}\text { Conocimiento, análisis } \\
\text { e investigación de los } \\
\text { contextos sociales y } \\
\text { educativos }\end{array}$ & $\begin{array}{l}\text { - Capacidad para detectar las necesidades educativas de un contexto determinado. } \\
\text { - Dominio de los planes de desarrollo de la comunidad y desarrollo local. } \\
\text { - Dominio de métodos, estrategias y técnicas de análisis de contextos socioeducativos. } \\
\text { - Pericia para discriminar las posibles respuestas educativas a necesidades, diferencián- } \\
\text { dolas de otros tipos de respuestas posibles (asistenciales, sanitarias, terapéuticas, etc.). } \\
\text { - Conocimiento y aplicación de los diversos marcos legislativos que posibilitan, orien- } \\
\text { tan y legitiman las acciones del Educador y la Educadora Social. } \\
\text { - Capacidad de análisis y evaluación del medio social y educativo (análisis de la } \\
\text { realidad). } \\
\text { - Conocimiento de las diferentes políticas sociales, educativas y culturales. }\end{array}$ \\
\hline $\begin{array}{l}\text { Diseño, implementa- } \\
\text { ción y evaluación de } \\
\text { programas y proyectos } \\
\text { en cualquier contexto } \\
\text { educativo. }\end{array}$ & $\begin{array}{l}\text { - Capacidad para formalizar los documentos básicos que regulan la acción socioedu- } \\
\text { cativa: proyecto de centro, reglamento de régimen interno, plan de trabajo, proyecto } \\
\text { educativo individualizado y otros informes socioeducativos. } \\
\text { - Dominio de técnicas de planificación, programación y diseño de programas y/o } \\
\text { proyectos. } \\
\text { - Capacidad de poner en marcha planes, programas, proyectos educativos y acciones } \\
\text { docentes. } \\
\text { - Conocimiento de las diversas técnicas/métodos de evaluación. }\end{array}$ \\
\hline $\begin{array}{l}\text { Gestión, dirección, co- } \\
\text { ordinación y organiza- } \\
\text { ción de instituciones y } \\
\text { recursos educativos. }\end{array}$ & $\begin{array}{l}\text { - Dominar los distintos modelos, técnicas y estrategias de dirección de programas, } \\
\text { equipamientos y recursos humanos. } \\
\text { - Destreza en gestión de proyectos, programas, centros y recursos educativos. } \\
\text { - Capacidad para la organización y gestión educativa de entidades e instituciones de } \\
\text { carácter social y/o educativo. } \\
\text { - Capacidad de supervisar el servicio ofrecido respecto a los objetivos marcados. } \\
\text { - Dominio en técnicas y estrategias de difusión de los proyectos. }\end{array}$ \\
\hline
\end{tabular}

Fuente: Asociación Estatal de Educación Social - ASEDES $(2007,46)$. 
Pensar en las competencias no nos dota de las mismas, pues adquirirlas es un proceso mucho más complejo y profundo. Es asumir que necesitamos ir más allá de la reproducción básica del conocimiento, técnicas o procedimientos que hemos ido adquiriendo.

Es comprender que, además de realizar las tareas propias de la profesión, se trata de que seamos capaces de resolver, en los diferentes posibles contextos, tareas mentales y prácticas complejas.

\section{LA FORMACIÓN DEL EDUCADOR SOCIAL EN ES- PAÑA DENTRO DEL MARCO EUROPEO}

La formación en Educación Social en España ha ido evolucionando a lo largo del tiempo paralelamente a su fundamentación teórico-científica y a la especialización de sus ámbitos o sectores de intervención. Tal y como indican Pereira y Solé (2013, 239) "se trata de una profesión en constante crecimiento". Si bien, para comprender el estado actual en el que se encuentra la formación de educadores sociales en España es necesario tener en consideración el marco europeo en el que se desenvuelve la educación superior. En este sentido, hace quince años tuvo lugar el acontecimiento más relevante de la reciente historia del Espacio Europeo de Educación Superior (en adelante, EEES). La Declaración de Bolonia (1999).

Durante este tiempo, las instituciones universitarias europeas, y por ende, las españolas, se han enfrentado a un escenario en el que el modelo universitario clásico se ha visto superado debido, principalmente, por la actuación de diversos acontecimientos mundiales. Entre otros: el crecimiento demográfico, el aumento de la renta per cápita, la aparición de países recientemente industrializados y con economías competitivas, el envejecimiento de la población y, por supuesto, la llegada de la crisis económica (DE-JUANAS y BELTRÁN, 2012 y 2014).
En los diez primeros años tras la Declaración de Bolonia, se establecieron y asentaron los elementos estructurales del EEES (DE-JUANAS, 2010). Estos elementos han resultado claves para la formación de los educadores sociales. El primer elemento fue la organización del currículum universitario en base a un Sistema Europeo de Transferencia de Créditos. Este sistema fue promovido sobre los planteamientos del programa ERASMUS-SÓCRATES en el que ya se utilizó un mecanismo para dar respuesta a las dificultades surgidas por convalidaciones en los diferentes intercambios de estudiantes (DIESTRO, 2010). Este sistema pretende que los diferentes estudios realizados en cualquier institución universitaria europea fueran reconocidos dentro de los márgenes del EEES. Para el caso concreto de la Educación Social, este hecho constituye un acontecimiento sin precedentes pues garantiza la movilidad de los estudiantes de esta titulación dentro de la Unión Europea.

El segundo elemento fue el establecimiento de una estructura común por ciclos en los estudios universitarios. Este elemento garantizó que la formación en Educación Social se ajustase a la realidad laboral de un modo óptimo, atendiendo a criterios de flexibilidad, equivalencia y reconocimiento de títulos en el marco de la Unión Europea. De tal modo, los estudios en Educación Social se ajustaron a una arquitectura compartida de titulaciones común a todos los europeos.

Esa estructura se sustenta básicamente en dos niveles. El primero, es el llamado Grado (Bachelor) que proporciona una titulación con un marcado carácter profesional y es condición fundamental para acceder a un segundo nivel. En España, la formación en educación social gira, principalmente, en torno a este primer nivel de grado que tiene una duración mínima de cuatro cursos académicos. El segundo nivel, Postgrado (Master), tiene una duración comprendida entre uno y dos años. En el caso de los graduados en Educación Social estas titulaciones ofrecen, por un lado, una mayor especialización profesional y, por otro, una formación para la investigación que dirige al 
estudiante hacía un nivel superior o tercer ciclo enfocado a los estudios propios de doctorado. En España y en Europa existen diferentes estudios de Master para los Educadores Sociales. Estas titulaciones suelen ofertarse en diferentes universidades y tienen diferentes enfoques. Uno de los estudios de Master con más demanda y con un enfoque profesional marcado es el Master en Formación del Profesorado de Educación Secundaria.

En este sentido, los educadores sociales, mediante su titulación pueden acceder a diferentes especialidades del Master según los criterios de admisión de las universidades que lo ofrecen. Por lo general, desde el Grado en Educación Social se puede cursar, en primer lugar la especialidad de Orientación Educativa y, en segundo lugar, la especialidad de Intervención Socio-comunitaria. En otro orden, se encuentra un título oficial de Master especialmente relevante para los educadores sociales como es el Master en Intervención Educativa en Contextos Sociales que se imparte en la Universidad Nacional de Educación a Distancia. Estos estudios siguen un doble enfoque, de investigación y profesional. En su desarrollo, este Master se vertebra en tres itinerarios o especialidades: Animación e Intervención Sociocultural; Calidad de vida en personas adultas y mayores; y, finalmente, Intervención con menores y jóvenes en dificultad social.

Para poder desarrollar toda esta arquitectura de niveles (grado, postgrado), se ha impuesto un diseño cíclico que viene determinado por el perfil profesional de cada titulación. En el caso de Educación Social se hizo necesario detallar las características propias de cada grupo profesional y definir los aprendizajes necesarios para que el educador social fuese competente en su actividad profesional. Con todo, se establecieron currículos basados en competencias en base a modelos de referencia que realmente aseguraron que se trabaja de un modo colaborativo y dentro de la comunidad.

El tercer elemento es el Suplemento al Diploma. Se trata de un documento adjunto al título que aporta información sobre la formación adquirida por un titulado y sus competencias profesionales. Este documento no pretende sustituir el título si no complementario y hacerlo más comprensible para poder comparar entre diferentes instituciones académicas. Este documento surgió para facilitar la libre circulación de los titulados por toda Europa.

El cuarto elemento son los sistemas de acreditación. Tras la Declaración de Bolonia, los diferentes países miembros de la Unión Europea se comprometieron a garantizar la calidad de los estudios universitarios mediante un conjunto de procedimientos y pautas que se encuentran bajo el amparo de la European Association for Quality Assurance in Higher Education (en adelante, ENQA). Esta agencia se propuso ofrecer a las instituciones universitarias instrumentos para evaluar su garantía interna y criterios para garantizar la calidad de sus estudios. En España, existen la Agencia Nacional de Evaluación de la Calidad y Acreditación y diferentes agencias autonómicas que supervisan a las instituciones y titulaciones universitarias bajo las pautas que ofrece la ENQA.

Finalmente, el quinto elemento son las titulaciones conjuntas que desde los comienzos del llamado Proceso de Bolonia se presentaron como un instrumento de promoción para la cooperación a nivel inter-institucional en Enseñanza Superior y fomentar la dimensión europea de la educación superior. Si bien, con el paso de los años, las titulaciones conjuntas no se han desarrollado por completo.

Desde la llegada del EEES, la enseñanza de la Educación Social se ha orientado hacia una formación profesional que aborda diferentes ámbitos en base a competencias (FULLANA, PALLISERA y PLANAS, 2011). Por ello, desde la comunidad académica existe un debate en constante desarrollo sobre dos posiciones que tienden a encontrarse y alejarse en proporciones similares: la primera, la construcción del currículo a partir de las necesidades reales de los actores de campo laboral; la segunda, la construcción del 
currículo a partir de las aportaciones del campo académico científico que por un lado aporta sus conocimientos teóricos y, por otro, aporta sus conocimientos por y para la investigación en la práctica educativa.

En ese sentido, las titulaciones encuentran un punto de encuentro para estas dos posiciones, se trata del Prácticum. Mediante la promoción y potenciación del Prácticum, los estudiantes que se encuentran en un periodo de formación inicial entran en contacto directo con la profesión y los diversos ámbitos laborales (PEREIRA y SOLÉ, 2013). Este periodo permite que los estudiantes conozcan diferentes salidas profesionales y puedan mejorar su cualificación.

\section{CONCLUSIONES}

En España, los ámbitos, perspectivas y figuras profesionales de los educadores sociales, que tienen lugar en la actualidad, responden a las necesidades políticas y sociales que han ido surgiendo a lo largo de un periodo de transición política que tuvo lugar dentro del propio país hasta un periodo de ajuste reciente ya en el marco de la Unión Europea. Con todo, en los últimos años, el educador social es una figura profesional que ejerce su labor en los servicios sociales desde dónde se atiende a la familia, infancia y juventud; así como en otros ámbitos relacionados con la animación sociocultural, educación de adultos y mayores.

En otro orden, durante los últimos cuarenta años, la figura del educador social se ha ido consolidando gracias a la creación de organismos colegiados que velan por mantener, fomentar e impulsar el desarrollo de propuestas asociativas entre educadores sociales.

Finalmente, la preocupación por la figura del educador social se ha trasladado a las entidades encargadas de su formación inicial y continúa. Así como a aquellas entidades y organismos nacionales e internacionales que velan por asegurar la calidad de la formación de los educadores sociales dentro de un marco regulador europeo. En este escenario, cobran especial relevancia las competencias profesionales que deben adquirir los diferentes profesionales de la Educación Social y la Pedagogía Social para atender a las demandas específicas de cada situación.

\section{REFERENCIAS}

A.S.E.D.E.S. Documentos profesionalizadores. Barcelona: Asociación Estatal de Educación Social (ASEDES), 2007.

BERMEJO, Lourdes (Coord.). Documento de consenso sobre Competencias Comunes de los Profesionales de la Gerontología. Madrid: Sociedad Española de Geriatría y Gerontología, 2013.

CARIDE, José Antonio. La Pedagogía Social en la transición democrática española: apuntes para una historia en construcción. In: Educació i Història: Revista d'Història de l'Educació, FR: 2011, n.18, p.37-59.

CHAMSEDDINE, Mohamed. Aproximación histórica a una de las profesionales sociales: La Educación Social. In: Revista de Educación Social, ES: 2013, n.17.

DE-JUANAS, Ángel. Contemplando Bolonia: una década de acontecimientos en la formación del EEES. In: Foro de Educación. FahrenHouse, ES: 2010, n.12, p.69-92.

DE-JUANAS, Ángel; BELTRÁN, Jesús. Creencias epistemológicas de los estudiantes de Pedagogía y Ciencias de la Educación. Revista de Psicodidáctica. UPV, ES: 2012, n.17, p.179-198.

DE-JUANAS, Ángel; BELTRÁN, Jesús. Valoraciones de los estudiantes de ciencias de la educación sobre la calidad de la docencia universitaria. In: Educación XXI: Revista de la Facultad de Educación. UNED, ES: 2014 , n.17, p.59-82. 
DIESTRO, Alfonso. El papel de la Conferencia permanente de ministros europeos de la educación en el establecimiento de una política educativa europea. In: Foro de Educación. FahrenHouse, ES: 2010, n.12, p.45-68.

FERNÁNDEZ, Jesús Damián y LIMÓN, Rosario. El arte de envejecer con humor. Málaga: Aljibe, 2012.

FULLANA, Judit; PALLISERA, María; PLANAS, Anna. Las competencias profesionales de los educadores sociales como punto de partida para el diseño curricular de la formación universitaria. Un estudio mediante el método Delphi. Revista Iberoamericana de Educación/Revista Ibero-americana de Educação. OEI, ES: 2011, n.56.

LIMÓN, Rosario. La pedagogía social como disciplina académica. Revista Educadores. ES: 1992, n.34, p. 233-246.

MELENDRO, Miguel. L' educatore sociale e l'intervento socioeducativo con i minori in Spagna. Pianeta Infancia, FR: 1999, n.12, p.269-279.
ORTEGA, José, CARIDE, José Antonio; ÚCAR, Xavier. La Pedagogía Social en la formación-profesionalización de los educadores y las educadoras sociales, o de cuando el pasado construye futuros. In: Revista de Educación Social, ES: 2013, n. 17.

PEREIRA, Carmen; SOLÉ, Jordi. La cualificación profesional en educación social. el papel del prácticum. Pedagogía Social. Revista interuniversitaria. SIPS, ES: 2013, n.21, p.237-258.

PÉREZ SERRANO, Gloria. Pedagogía Social-Educación Social. Construcción científica e intervención práctica. Madrid: Narcea, 2004.

SÁEZ CARRERAS, Juan. El enfoque por competencias en la formación de los educadores sociales: una mirada a su caja de herramientas. Pedagogía Social. Revista interuniversitaria. SIPS, ES: 2009, n.16, p.9-20.

TIANA, Alejandro; SOMOZA, José Miguel y BADANELLI, Ana. Historia de la Educación Social. Madrid: UNED, 2014.

1. Profesor Doctor del Departamento de Teoría de la Educación y Pedagogía Social de la Universidad Nacional de Educación a Distancia. E-mail: adejuanas@edu.uned.es

2. Profesora Doctora de la Faculdad de Educación de la Universidad Complutense de Madrid. Facultad de Educación de la Universidad Complutense de Madrid. Departamento de Teoría e Historia de la Educación. E-mail: mrlimonm@edu.ucm.es

3. Profesor Doctor del Departamento de Teoría de la Educación y Pedagogía Social de la Universidad Nacional de Educación a Distancia. E-mail: mmelendro@edu.uned.es 